\title{
SPSS and SAS programs for comparing Pearson correlations and OLS regression coefficients
}

\author{
Bruce Weaver • Karl L. Wuensch
}

Published online: 24 January 2013

(C) Psychonomic Society, Inc. 2013

\begin{abstract}
Several procedures that use summary data to test hypotheses about Pearson correlations and ordinary least squares regression coefficients have been described in various books and articles. To our knowledge, however, no single resource describes all of the most common tests. Furthermore, many of these tests have not yet been implemented in popular statistical software packages such as SPSS and SAS. In this article, we describe all of the most common tests and provide SPSS and SAS programs to perform them. When they are applicable, our code also computes $100 \times(1-\alpha) \%$ confidence intervals corresponding to the tests. For testing hypotheses about independent regression coefficients, we demonstrate one method that uses summary data and another that uses raw data (i.e., Potthoff analysis). When the raw data are available, the latter method is preferred, because use of summary data entails some loss of precision due to rounding.
\end{abstract}

Keywords Correlation $\cdot$ Regression $\cdot$ Ordinary least squares $\cdot$ SPSS $\cdot$ SAS

Electronic supplementary material The online version of this article (doi:10.3758/s13428-012-0289-7) contains supplementary material, which is available to authorized users.

\section{B. Weaver}

Human Sciences Division, Northern Ontario School of Medicine, Thunder Bay, ON, Canada P7B 5E1

B. Weaver $(\bowtie)$

Centre for Research on Safe Driving, Lakehead University,

Thunder Bay, ON, Canada P7B 5E1

e-mail: bweaver@lakeheadu.ca

K. L. Wuensch

Department of Psychology, East Carolina University, Greenville, NC, USA 27858-4353

\section{Introduction}

Several textbooks and articles describe methods for testing hypotheses concerning Pearson correlations and coefficients from ordinary least squares (OLS) regression models (e.g., Howell, 2013; Kenny, 1987; Potthoff, 1966; Raghunathan, Rosenthal, \& Rubin, 1996; Steiger, 1980). However, we are not aware of any single resource that describes all of the most common procedures. Furthermore, many of the methods described in those various resources have not yet been implemented in standard statistical software packages such as SPSS and SAS. In some cases, data analysts may find stand-alone programs that perform the desired tests. ${ }^{1}$ However, such programs can be relatively difficult to use (e.g., if they are old 16-bit DOS programs, they may not run on modern computers), or they may not provide all of the desired output (e.g., one program we found reports a $z$-test result, but not the corresponding $p$-value). It would be much more convenient if one could carry out all of these tests using one's usual statistical software. With that in mind, the twofold purpose of this article is to provide a single resource that briefly reviews the most common methods for testing hypotheses about Pearson correlations and OLS regression coefficients and to provide SPSS and SAS code that performs the calculations. When they are applicable, our code also computes $100 \times(1-\alpha) \%$ confidence intervals (CIs) corresponding to the statistical tests.

We describe the various methods in this order: methods concerning (1) single parameters (e.g., testing the significance of a correlation), (2) two independent parameters (e.g., the difference between two independent correlations), (3) $k$ independent parameters, where $k \geq 2$ (e.g., testing the

\footnotetext{
${ }^{1}$ For example, James Steiger's Multicorr program (http://www.statpower. net/Software.html) can be used to perform "single sample comparisons of correlations"; and Calvin Garbin's FZT program (http://psych.unl.edu/ psycrs/statpage/comp.html) can be used to compute "a variety of $r$ and $R^{2}$ comparison tests."
} 
equivalence of three correlations), and (4) two nonindependent parameters (e.g., the difference between two nonindependent correlations). In all cases, SPSS and SAS programs to carry out the computations are provided as part of the online supplementary material, along with the output they generate. (The data files, code, and output are also available on the authors' Web sites: https://sites.google.com/a/lakeheadu.ca/ bweaver/Home/statistics/spss/my-spss-page/weaver wuensch and http://core.ecu.edu/psyc/wuenschk/W\&W/ W\&W-SAS.htm.) Users can select the desired confidence level for CIs (when they are applicable) by setting the value of a variable called alpha (e.g., set alpha $=.05$ to obtain a $95 \%$ $\mathrm{CI}$, alpha $=.01$ to obtain a $99 \% \mathrm{CI}$, etc.).

To illustrate the various methods, we use the lung function data set from Afifi, Clark, and May's (2003) book Computer-Aided Multivariate Analysis. We chose this data set for two reasons: (1) It contains variables suitable for demonstrating all of the methods we discuss, and (2) readers can easily download it in several formats (SAS, Stata, SPSS, Statistica, S-Plus, and ASCII) from the UCLA Academic Technology Services Web site (http://www.ats.ucla.edu/stat/ spss/examples/cama4/default.htm). The data are from the UCLA study of chronic obstructive pulmonary disease. Afifi and coauthors described this file as "a subset including [nonsmoking] families with both a mother and a father, and one, two, or three children between the ages of 7 and 17 who answered the questionnaire and took the lung function tests at the first time period." The variables we use are area of the state (four levels) plus height (in inches) and weight (in pounds) for both fathers (variable names FHEIGHT and FWEIGHT) and mothers (MHEIGHT and MWEIGHT). Note that the initial $\mathrm{F}$ and $\mathrm{M}$ for the height and weight variables stand for father's and mother's, not female and male.

Input data for most of the code we provide consist of summary statistics that we computed using the lung function data. For example, we computed within each of the four different regions a correlation matrix for father's height, father's weight, mother's height, and mother's weight (variables FHEIGHT, FWEIGHT, MHEIGHT, and MWEIGHT). Table 1 shows those four correlation matrices. We also carried out some regression analyses, the results of which are displayed later in the article.

\section{Methods for single parameters}

Testing the null hypothesis that $\rho=$ a specified value

The correlation matrices shown in Table 1 include a $p$-value for each correlation. If those same correlations appeared in a report or article that did not include $p$-values, one could work out the $p$-values by computing a $t$-test on each of the Pearson $r$ values, as shown in Eq. 1. Under the null hypothesis that $\rho=0$, the test statistic $t$ is asymptotically distributed as $t$ with $d f=n-2:^{2}$

$t=\frac{r \sqrt{n-2}}{\sqrt{1-r^{2}}}$.

When a null hypothesis that specifies a nonzero value for $\rho$ is tested, things are more complicated. As Howell (2013) put it, "When $\rho \neq 0$, the sampling distribution of $r$ is not approximately normal (it becomes more and more skewed as $\rho \Rightarrow \pm 1.00$ ), and its standard error is not easily estimated" (p. 284). Fortunately, there is a straightforward solution to this problem: One can apply Fisher's (1921) $r$-to- $z$ transformation to both $r$ and $\rho$. Equation 2 shows the application of Fisher's transformation to $r$, and Eq. 3 shows the inverse transformation from $r^{\prime}$ to $r^{3}{ }^{3}$ Fisher showed that the sampling distribution of $r^{\prime}$ is approximately normal with variance equal to $1 /(n-3)$, where $n$ is the sample size. Taking the square root of that variance yields the standard error of $r^{\prime}$ (see Eq. 4):

$r^{\prime}=(0.5) \log _{e}\left|\frac{1+r}{1-r}\right|$

$r=\frac{e^{r^{\prime}}-e^{-r^{\prime}}}{e^{r^{\prime}}+e^{-r^{\prime}}}=\frac{e^{2 r^{\prime}}-1}{e^{2 r^{\prime}}+1}$

$s_{r^{\prime}}=\sqrt{\frac{1}{n-3}}$.

The final step is to compute a $z$-test (see Eq. 5). The $p$ value for this z-test is obtained in the usual fashion (i.e., using the standard normal distribution). This $z$-test can be used even when the null hypothesis states that $\rho=0$ (and our

\footnotetext{
$\overline{2}$ The symbol $\rho$ is the Greek letter rho. It is used to represent the population correlation.

${ }^{3}$ Because the sampling distribution of the transformed value is approximately normal, Fisher (1921) called it $z$. Following Howell (2013) and many other authors, we call it $r^{\prime}$ instead, in order to avoid confusion with the $z$-test value to be reported shortly. (Some authors use $z_{r}$ rather than $z$, for the same reason.)
} 
Table 1 Pearson correlations computed using the height and weight variables for fathers and mothers in the lung function data file

\begin{tabular}{|c|c|c|c|c|c|c|}
\hline Area of state & & & $\begin{array}{l}\text { Height of father in } \\
\text { inches }\end{array}$ & $\begin{array}{l}\text { Weight of father in } \\
\text { pounds }\end{array}$ & $\begin{array}{l}\text { Height of mother } \\
\text { in inches }\end{array}$ & $\begin{array}{l}\text { Weight of mother } \\
\text { in pounds }\end{array}$ \\
\hline \multirow[t]{8}{*}{ Burbank $(\mathrm{n}=24)$} & \multirow[t]{2}{*}{ Height of father in inches } & Pearson & 1 & $.628^{* *}$ & .164 & -.189 \\
\hline & & Sig. (2-tailed) & & .001 & .443 & .376 \\
\hline & \multirow[t]{2}{*}{ Weight of father in pounds } & Pearson & $.628^{* *}$ & 1 & -.145 & -.201 \\
\hline & & Sig. (2-tailed) & .001 & & .499 & .346 \\
\hline & \multirow[t]{2}{*}{ Height of mother in inches } & Pearson & .164 & -.145 & 1 & $.624^{* *}$ \\
\hline & & Sig. (2-tailed) & .443 & .499 & & .001 \\
\hline & \multirow[t]{2}{*}{ Weight of mother in pounds } & Pearson & -.189 & -.201 & $.624^{* *}$ & 1 \\
\hline & & Sig. (2-tailed) & .376 & .346 & .001 & \\
\hline \multirow[t]{8}{*}{ Lancaster $(\mathrm{n}=49)$} & \multirow[t]{2}{*}{ Height of father in inches } & Pearson & 1 & $.418^{* *}$ & .198 & .065 \\
\hline & & Sig. (2-tailed) & & .003 & .172 & .660 \\
\hline & \multirow[t]{2}{*}{ Weight of father in pounds } & Pearson & $.418^{* *}$ & 1 & -.181 & $.299^{*}$ \\
\hline & & Sig. (2-tailed) & .003 & & .214 & .037 \\
\hline & \multirow[t]{2}{*}{ Height of mother in inches } & Pearson & .198 & -.181 & 1 & .040 \\
\hline & & Sig. (2-tailed) & .172 & .214 & & .786 \\
\hline & \multirow[t]{2}{*}{ Weight of mother in pounds } & Pearson & .065 & $.299^{*}$ & .040 & 1 \\
\hline & & Sig. (2-tailed) & .660 & .037 & .786 & \\
\hline \multirow[t]{8}{*}{ Long Beach $(n=19)$} & \multirow[t]{2}{*}{ Height of father in inches } & Pearson & 1 & .438 & .412 & .114 \\
\hline & & Sig. (2-tailed) & & .061 & .079 & .641 \\
\hline & \multirow[t]{2}{*}{ Weight of father in pounds } & Pearson & .438 & 1 & -.032 & .230 \\
\hline & & Sig. (2-tailed) & .061 & & .898 & .343 \\
\hline & \multirow[t]{2}{*}{ Height of mother in inches } & Pearson & .412 & -.032 & 1 & $.487^{*}$ \\
\hline & & Sig. (2-tailed) & .079 & .898 & & .035 \\
\hline & \multirow[t]{2}{*}{ Weight of mother in pounds } & Pearson & .114 & .230 & $.487^{*}$ & 1 \\
\hline & & Sig. (2-tailed) & .641 & .343 & .035 & \\
\hline \multirow[t]{8}{*}{ Glendora $(n=58)$} & \multirow[t]{2}{*}{ Height of father in inches } & Pearson & 1 & $.589^{* *}$ & $.366^{* *}$ & .071 \\
\hline & & Sig. (2-tailed) & & .000 & .005 & .596 \\
\hline & \multirow[t]{2}{*}{ Weight of father in pounds } & Pearson & $.589^{* *}$ & 1 & $.330^{*}$ & .209 \\
\hline & & Sig. (2-tailed) & .000 & & .011 & .115 \\
\hline & \multirow[t]{2}{*}{ Height of mother in inches } & Pearson & $.366^{* *}$ & $.330^{*}$ & 1 & $.364^{* *}$ \\
\hline & & Sig. (2-tailed) & .005 & .011 & & .005 \\
\hline & \multirow[t]{2}{*}{ Weight of mother in pounds } & Pearson & .071 & .209 & $.364^{* *}$ & 1 \\
\hline & & Sig. (2-tailed) & .596 & .115 & .005 & \\
\hline
\end{tabular}

code computes it), but in that case, the $t$-test shown in Eq. $1 \quad 100(1-\alpha) \%$ CI for $\rho^{\prime}=\mathrm{r}^{\prime} \pm \mathrm{z}_{\alpha / 2} \mathrm{~S}_{\mathrm{r}^{\prime}}$. is preferred.

Equation 6 shows how the standard error of $r^{\prime}$ (Eq. 4) can be used to compute a CI for $\rho^{\prime}$. The $z_{\alpha / 2}$ in Eq. 6 represents the critical value of $z$ for a two-tailed test with $\alpha$ set to the desired level. For a $95 \% \mathrm{CI}$, for example, $\alpha=.05$, and $z_{\alpha / 2}=1.96$. The inverse of the $r$-to- $z$ transformation (Eq. 3) is used to convert the lower and upper confidence limits for $\rho^{\prime}$ into confidence limits for $\rho$ :

$z=\frac{r^{\prime}-\rho^{\prime}}{s_{r^{\prime}}}=\frac{r^{\prime}-\rho^{\prime}}{\sqrt{\frac{1}{n-3}}}$
Whereas the choice of test statistic ( $t$ vs. $z$ ) depends on whether the null hypothesis specifies that $\rho=0$ versus some nonzero value, the computation of confidence limits for $\rho$ does not. The method shown in Eq. 6 is used to compute a CI regardless of the value of $\rho$ under the null hypothesis.

Our code for illustrating these methods requires the following input variables: $r$ (the observed Pearson $r$ ), rho (the population correlation according to $\mathrm{H}_{0}$ ), $\mathrm{n}$ (the sample size), alpha (the value used to determine the confidence level for the CI on rho), and Note, a text field in which a brief 
description of the data can be entered. The SPSS code for this situation has the following DATA LIST command ${ }^{4}$ :

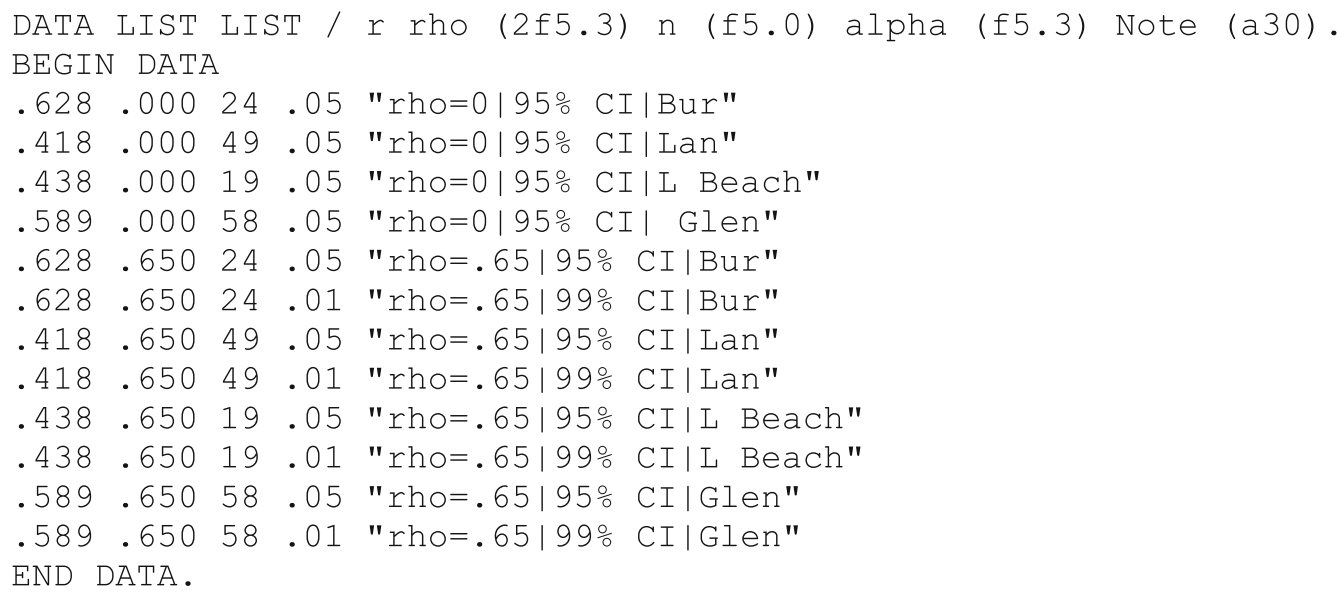

The correlations entered in variable $r$ are the correlations between father's height and father's weight for the four areas of the state (see Table 1). The first four rows of input set rho = 0 , whereas the last eight rows set rho $=.650 .^{5}$ Therefore, our code uses the $t$-test shown in Eq. 1 for only the first four rows, whereas the $z$-test in Eq. 5 is computed for every row. Note too that the value of alpha is .05 in some rows of input data and .01 in others. Our code computes $95 \%$ CIs where alpha $=.05$ and $99 \%$ CIs where alpha $=.01 .^{6}$ All CIs are computed via Eq. 6. The output from our SPSS code is listed below.

\begin{tabular}{|c|c|c|c|c|c|c|c|c|c|c|c|}
\hline$r$ & rho & $\mathrm{n}$ & $t$ & $d f$ & $p_{-} t$ & $\mathrm{z}$ & $P_{-} z$ & alpha & Lower & Upper & Note \\
\hline .628 & .000 & 24 & 3.785 & 22 & .001 & 3.382 & .001 & .050 & .301 & .823 & $r h o=0 \mid 95 \%$ CI $\mid$ Bur \\
\hline .418 & .000 & 49 & 3.154 & 47 & .003 & 3.020 & .003 & .050 & .155 & .626 & $r h o=0 \mid 95 \%$ CI I Lan \\
\hline .438 & .000 & 19 & 2.009 & 17 & .061 & 1.879 & .060 & .050 & -.020 & .744 & $r h o=0 \mid 95 \%$ CIIL Beach \\
\hline .589 & .000 & 58 & 5.454 & 56 & .000 & 5.014 & .000 & .050 & .390 & .735 & rho $=0 \mid 95 \%$ CII Glen \\
\hline .628 & .650 & 24 & . & $\cdot$ & . & -.170 & .865 & .050 & .301 & .823 & rho $=.65 \mid 95 \%$ CI |Bur \\
\hline .628 & .650 & 24 & . & . & . & -.170 & .865 & .010 & .174 & .862 & rho $=.65 \mid 99 \%$ CI|Bur \\
\hline .418 & .650 & 49 & . & - & . & -2.238 & .025 & .050 & .155 & .626 & rho $=.65 \mid 95 \%$ CI |Lan \\
\hline .418 & .650 & 49 & - & • & - & -2.238 & .025 & .010 & .065 & .678 & rho $=.65 \mid 99 \%$ CI |Lan \\
\hline .438 & .650 & 19 & . & . & . & -1.222 & .222 & .050 & -.020 & .744 & rho $=.65 \mid 95 \%$ CI|L Beach \\
\hline .438 & .650 & 19 & - & - & - & $-1 \cdot 222$ & .222 & .010 & -.172 & .805 & rho $=.65 \mid 99 \%$ CI/L Beach \\
\hline .589 & .650 & 58 & . & . & . & -.735 & .462 & .050 & .390 & .735 & rho $=.65 \mid 95 \%$ CI|Glen \\
\hline .589 & .650 & 58 & . & • & . & -.735 & .462 & .010 & .317 & .771 & rho $=.65|99 \% \mathrm{CI}| \mathrm{Glen}$ \\
\hline
\end{tabular}

The $t$-test results in the first four rows of output indicate that the correlation between height and weight (for fathers) is statistically significant in all four areas

\footnotetext{
${ }^{4}$ Users who wish to analyze their own data can do so by replacing the data lines between BEGIN DATA and END DATA and then running the syntax.

${ }^{5}$ We don't know the value of the actual population correlation between height and weight of the fathers. We chose .650 because it was convenient for producing a mix of significant and nonsignificant $z$-tests.
}

except area 3, Long Beach. Note too that the $p$-values for those correlations $(.001, .003, .060$, and .000) agree almost perfectly with the $p$-values reported in Table 1 . The only differences (e.g., .060 vs. .061 for Long Beach) are due to loss of precision resulting from our use of summary data.

\footnotetext{
${ }^{6}$ In general, our code computes CIs with confidence level $=100(1-\alpha) \%$.
} 
Table 2 Parameter estimates for four simple linear regression models with father's height regressed on father's weight; father's height was centered on 60 in. $(5 \mathrm{ft})$

\begin{tabular}{|c|c|c|c|c|c|c|c|c|}
\hline \multirow[t]{2}{*}{ Area } & & \multicolumn{2}{|c|}{$\begin{array}{l}\text { Unstandardized } \\
\text { coefficients }\end{array}$} & \multirow{2}{*}{$\begin{array}{l}\text { Standardized } \\
\text { coefficients } \\
\text { Beta }\end{array}$} & \multirow[t]{2}{*}{$\mathrm{t}$} & \multirow[t]{2}{*}{ Sig. } & \multicolumn{2}{|c|}{$95.0 \%$ confidence interval for $\mathrm{B}$} \\
\hline & & $\mathrm{B}$ & Std. error & & & & Lower bound & Upper bound \\
\hline \multirow[t]{2}{*}{ Burbank $(\mathrm{n}=24)$} & (Constant) & 142.011 & 10.664 & & 13.317 & .000 & 119.896 & 164.127 \\
\hline & $\begin{array}{l}\text { Height of father (centered on } \\
60 \text { in) }\end{array}$ & 4.179 & 1.105 & .628 & 3.781 & .001 & 1.887 & 6.472 \\
\hline \multirow[t]{2}{*}{ Lancaster $(n=49)$} & (Constant) & 148.053 & 11.142 & & 13.288 & .000 & 125.638 & 170.468 \\
\hline & $\begin{array}{l}\text { Height of father (centered on } \\
60 \text { in) }\end{array}$ & 3.709 & 1.177 & .418 & 3.151 & .003 & 1.341 & 6.078 \\
\hline \multirow{2}{*}{$\begin{array}{l}\text { Long Beach } \\
\quad(n=19)\end{array}$} & (Constant) & 144.038 & 18.250 & & 7.893 & .000 & 105.535 & 182.541 \\
\hline & $\begin{array}{l}\text { Height of father (centered on } \\
60 \text { in) }\end{array}$ & 3.749 & 1.866 & .438 & 2.009 & .061 & -.187 & 7.685 \\
\hline \multirow[t]{2}{*}{ Glendora $(\mathrm{n}=58)$} & (Constant) & 130.445 & 10.228 & & 12.753 & .000 & 109.955 & 150.935 \\
\hline & $\begin{array}{l}\text { Height of father (centered on } \\
60 \text { in) }\end{array}$ & 5.689 & 1.044 & .589 & 5.451 & .000 & 3.598 & 7.780 \\
\hline
\end{tabular}

${ }^{\mathrm{a}}$ Dependent Variable: weight of father in pounds

In the final eight rows of output, where $\rho=.650$ under the null hypothesis, only the $z$-test is computed. Note that we included the input data for each area twice, first with alpha $=.05$ and again with alpha $=$ .01 . Thus, the first line of output for each area displays a $95 \% \mathrm{CI}$, and the second a $99 \% \mathrm{CI}$. The $z$-test result is unaffected by the value of alpha, which is why the same test result appears twice for each area. Only in area 1 (Lancaster) does the observed correlation differ significantly from $.650, z=-2.238, p=.025$.

Testing the hypothesis that $b=$ a specified value

The data we use as an illustration in this section come from four simple linear regression models (one for each area) with father's weight regressed on father's height. In order to make the intercepts more meaningful, we first centered height on 60 in. $(5 \mathrm{ft}){ }^{7}$ Parameter estimates for the four models are shown in Table 2.

In his discussion of this topic, Howell (2013) began by showing that the standard error of $b\left(s_{b}\right)$ can be computed from the standard error of $Y$ given $X\left(s_{Y \mid X}\right)$, the standard deviation of the $X$ scores $\left(s_{X}\right)$, and the sample size $(n)$. Given

\footnotetext{
${ }^{7}$ In other words, we used a transformed height variable equal to height minus $60 \mathrm{in}$. If we had used the original height variable, the constant from our model would have given the fitted value of weight when height $=0$, which would be nonsensical. With height centered on 60 in., the constant gives the fitted value of weight when height $=$ 60 in.
}

that $s_{Y \mid X}=\sqrt{M S_{\text {error }}}$, or the root mean square error (RMSE), $s_{b}$ can be computed as shown in Eq. 7:

$s_{b}=\frac{R M S E}{s_{X} \sqrt{n-1}}=\frac{R M S E}{\sqrt{S S_{X}}}$.

However, it is extremely difficult to imagine circumstances under which one would have the RMSE from the regression model (plus the sample size and the standard deviation of $X$ ), but not the standard error of $b$. Therefore, we do not provide code to compute the standard error of $b$ as shown in Eq. 7. Instead, we simply take the standard error of $b$ from the regression output and plug it into Eq. 8, which shows a $t$-test for the null hypothesis that $b^{*}$, the population parameter corresponding to $b$, is equal to a specified value. ${ }^{8}$ The $m$ in the subscript is the number of predictor variables, not including the constant, and $n-m-1$ equals the degrees of freedom for the $t$-test. ${ }^{9}$ The standard error of $b$ is also used to compute a $100(1-\alpha) \%$ CI for $b^{*}$ (Eq. 9):

$t_{n-m-1}=\frac{b-b^{*}}{s_{b}}$

$100(1-\alpha) \% \mathrm{CI}$ for $\mathrm{b}^{*}=\mathrm{b} \pm \mathrm{t}_{\alpha / 2} \mathrm{~S}_{\mathrm{b}}$.

\footnotetext{
${ }^{8}$ We follow Howell (2013) in using $b^{*}$ rather than $\beta$ to represent the parameter corresponding to $b$. We do this to avoid "confusion with the standardized regression coefficient," which is typically represented by $\beta$.

${ }^{9}$ Although some authors use $p$ to represent the number of predictors in a regression model, we use $m$ in this context in order to avoid confusion with the $p$-value.
} 
As we saw earlier, when testing hypotheses about $\rho$, we can use the $t$-test shown in Eq. 1 when the null hypothesis states that $\rho=0$; but when the null hypothesis states that $\rho=$ some nonzero value, we must apply Fisher's $r$-to- $z$ transformation to both $r$ and $\rho$ and then use the $z$-test shown in Eq. 5. For regression coefficients, on the other hand, the $t$ - test shown in Eq. 8 can be used regardless of the value of $b^{*}$. In other words, when $b^{*}=0$, we will get the usual $t$-test shown in the table of regression coefficients. To confirm this, we plugged the displayed values of the intercept and slope into our implementation of Eq. 8 and set $b^{*}=0$. Doing so produced the following output:

\begin{tabular}{|c|c|c|c|c|c|c|c|c|c|}
\hline $\mathrm{b}$ & bstar & se & $t$ & $d f$ & $\mathrm{p}$ & alpha & CI_Lower & CI_Upper & Note \\
\hline 142.011 & .000 & 10.664 & 13.317 & 22 & .000 & .050 & 119.895 & 164.127 & Int, Bur \\
\hline 148.053 & .000 & 11.142 & 13.288 & 47 & .000 & .050 & 125.638 & 170.468 & Int, Lan \\
\hline 144.038 & .000 & 18.250 & 7.892 & 17 & .000 & .050 & 105.534 & 182.542 & Int, L Beach \\
\hline 130.445 & .000 & 10.228 & 12.754 & 56 & .000 & .050 & 109.956 & 150.934 & Int, Glen \\
\hline 4.179 & .000 & 1.105 & 3.782 & 22 & .001 & .050 & 1.887 & 6.471 & Slope, Bur \\
\hline 3.709 & .000 & 1.177 & 3.151 & 47 & .003 & .050 & 1.341 & 6.077 & Slope, Lan \\
\hline 3.749 & .000 & 1.866 & 2.009 & 17 & .061 & .050 & -.188 & 7.686 & Slope, L Beach \\
\hline 5.689 & .000 & 1.044 & 5.449 & 56 & .000 & .050 & 3.598 & 7.780 & Slope, Glen \\
\hline
\end{tabular}

Apart from some rounding error, the results of these $t$ tests match those shown in Table 2. Note that alpha $=.05$ on every line, so all CIs are $95 \%$ CIs.

Now suppose that we have reason to believe that the true population values for the intercept and slope are
145 and 3.5, respectively, and we wish to compare our sample values with those parameters. Plugging the observed intercepts and slopes into our SPSS implementation of Eq. 8 with $b^{*}=145$ for intercepts and $b^{*}=3.5$ for slopes, we get the output listed below:

\begin{tabular}{|c|c|c|c|c|c|c|c|c|c|}
\hline $\mathrm{b}$ & bstar & se & $t$ & $d f$ & $\mathrm{p}$ & alpha & CI_Lower & CI_Upper & Note \\
\hline 142.011 & 145.000 & 10.664 & -.280 & 22 & .782 & .050 & 119.895 & 164.127 & Int, Bur \\
\hline 148.053 & 145.000 & 11.142 & .274 & 47 & .785 & .050 & 125.638 & 170.468 & Int, Lan \\
\hline 144.038 & 145.000 & 18.250 & -.053 & 17 & .959 & .050 & 105.534 & 182.542 & Int, L Beach \\
\hline 130.445 & 145.000 & 10.228 & -1.423 & 56 & .160 & .050 & 109.956 & 150.934 & Int, Glen \\
\hline 4.179 & 3.500 & 1.105 & .614 & 22 & .545 & .050 & 1.887 & 6.471 & Slope, Bur \\
\hline 3.709 & 3.500 & 1.177 & .178 & 47 & .860 & .050 & 1.341 & 6.077 & Slope, Lan \\
\hline 3.749 & 3.500 & 1.866 & .133 & 17 & .895 & .050 & -.188 & 7.686 & Slope, I Beach \\
\hline 5.689 & 3.500 & 1.044 & 2.097 & 56 & .041 & .050 & 3.598 & 7.780 & Slope, Glen \\
\hline
\end{tabular}

Looking first at the results for the intercepts, we would fail to reject the null hypothesis (that $b^{*}=145$ ) in all four cases, because all $p$-values are greater than .05. For the slopes, on the other hand, we would reject the null hypothesis (that $b^{*}=3.5$ ) for Glendora, $t(56)=2.097, p=.041$, but not for any of the other three areas (where all $t$-ratios are $<1$ and all $p$-values are $\geq .545$ ).

\section{Methods for two independent parameters}

We now shift our focus to tests and CIs for the difference between two independent parameters.
Testing the difference between two independent correlations

When the correlation between two variables is computed in two independent samples, one may wish to test the null hypothesis that the two population correlations are the same $\left(H_{0}: \rho_{1}=\rho_{2}\right)$. To test this null hypothesis, we use a simple extension of the method for testing the null that $\rho=$ a specified value. As in that case, we must apply Fisher's $r$-to- $z$ transformation to convert the two sample correlations into $r^{\prime}$ values. As is shown in Eq. 4, the standard error of an $r^{\prime}$ value is $\sqrt{1 /(n-3)}$. Squaring that expression (i.e., removing the square root sign) gives the variance of the 
sampling distribution of $r^{\prime}$. The variance of the difference between two independent $r^{\prime}$ values is the sum of their variances. ${ }^{10}$ Taking the square root of that sum of variances yields the standard error of the difference between two independent $r^{\prime}$ values (see Eq. 10). That standard error is used as the denominator in a $z$-test (see Eq. 11):

$$
s_{r_{1}^{\prime}-r_{2}^{\prime}}=\sqrt{\frac{1}{n_{1}-3}+\frac{1}{n_{2}-3}}
$$

$z=\frac{r_{1}^{\prime}-r_{2}^{\prime}}{s_{r_{1}^{\prime}-r_{2}^{\prime}}^{\prime}}=\frac{r_{1}^{\prime}-r_{2}^{\prime}}{\sqrt{\frac{1}{n_{1}-3}+\frac{1}{n_{2}-3}}}$.

We illustrate these computations using several independent pairs of correlations from Table $1 .{ }^{11}$ In each case, we compare the values for Lancaster and Glendora, the two areas with the largest sample sizes. Plugging the needed values into our implementation of Eq. 11 gave us the output shown below:

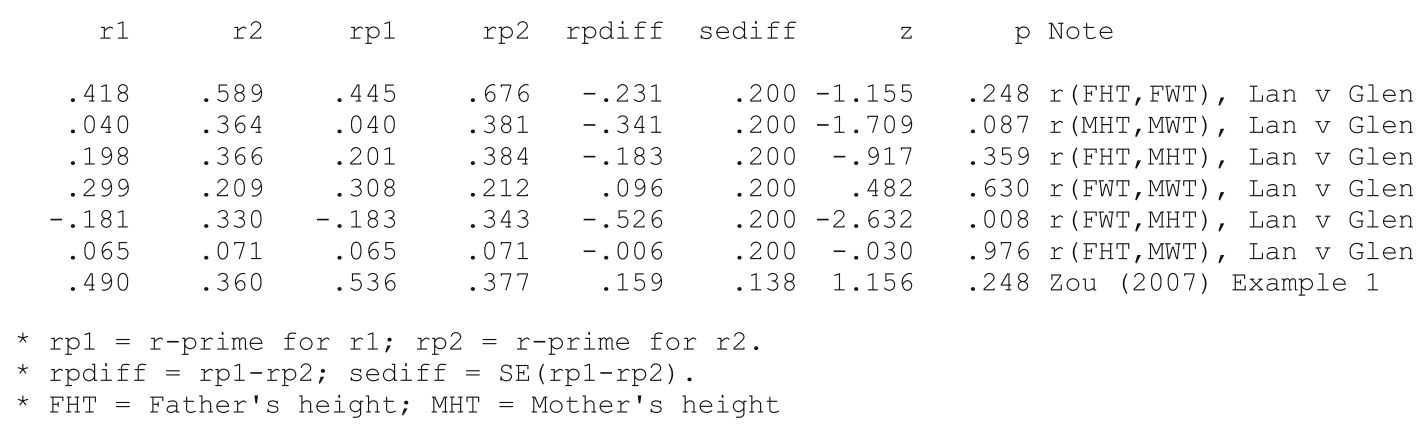

In the Note column, the initial $\mathrm{F}$ and $\mathrm{H}$ stand for father's and mother's respectively, and HT and WT stand for height and weight. Thus, the r(FHT,FWT) on the first line indicates that the correlation between father's height and father's weight has been computed for both Lancaster and Glendora and the two correlations have been compared. The $r p 1$ and $r p 2$ columns give the $r^{\prime}$ values corresponding to $r_{1}$ and $r_{2}$. (Standard errors for $r p 1$ and $r p 2$ are also computed but are not listed here, in order to keep the output listing to a manageable width.) The rpdiff and sediff columns show the numerator and denominator of Eq. 11. The null hypothesis (that $\rho_{1}-\rho_{2}=0$ ) can be rejected only for the test comparing the correlations between father's weight and mother's height, $z=-2.632, p=.008$. For all other comparisons, the $p$-values are greater than .05 .

Our code also computes $100 \times(1-\alpha) \%$ CIs for $\rho_{1}$, $\rho_{2}$, and $\rho_{1}-\rho_{2}$. CIs for $\rho_{1}$ and $\rho_{2}$ are obtained by

\footnotetext{
${ }^{10}$ More generally, the variance of the difference is the sum of the variances minus two times the covariance. But when the samples are independent, the covariance is equal to zero.
}

computing CIs for $\rho^{\prime}{ }_{1}$ and $\rho_{2}^{\prime}{ }_{2}$ (see Eq. 6) and then back-transforming them (Eq. 3). The CI for $\rho_{1}-\rho_{2}$ is computed using Zou's (2007) modified asymptotic (MA) method. ${ }^{12}$ The first listing below shows CIs for $\rho_{1}$ and $\rho_{2}$, and the second listing shows the CI for $\rho_{1}-\rho_{2}$. (We included Zou's example in order to verify that our code for his method was correct.) Alpha $=.05$ in all cases, so they are all $95 \%$ CIs.

\footnotetext{
${ }^{11}$ Readers may wonder why we do not compare the correlation between height and weight for fathers with the same correlation for mothers. Given that there are matched pairs of fathers and mothers, those correlations are not independent. Therefore, it would be inappropriate to use this method for comparing them. However, we do compare those two correlations later, using the $Z P F$ statistic, which takes into account the dependency.

${ }^{12}$ We use Zou's MA method because his simulations demonstrate that for the situations listed below, it provides coverage much closer (on average) to the nominal confidence level than do the more conventional methods: (1) comparing two independent correlations, (2) comparing two correlated correlations with one variable in common, and (3) comparing two correlated correlations with no variables in common.
} 
Fig. 1 The relationship between fathers' heights and weights in the Lancaster and Glendora samples (blue and red symbols respectively). Height was centered on 60 in.; therefore, the intercepts for the two models (148.053 and 130.45 ) occur at the intersections of the two regression lines with the dashed line at height $=60$



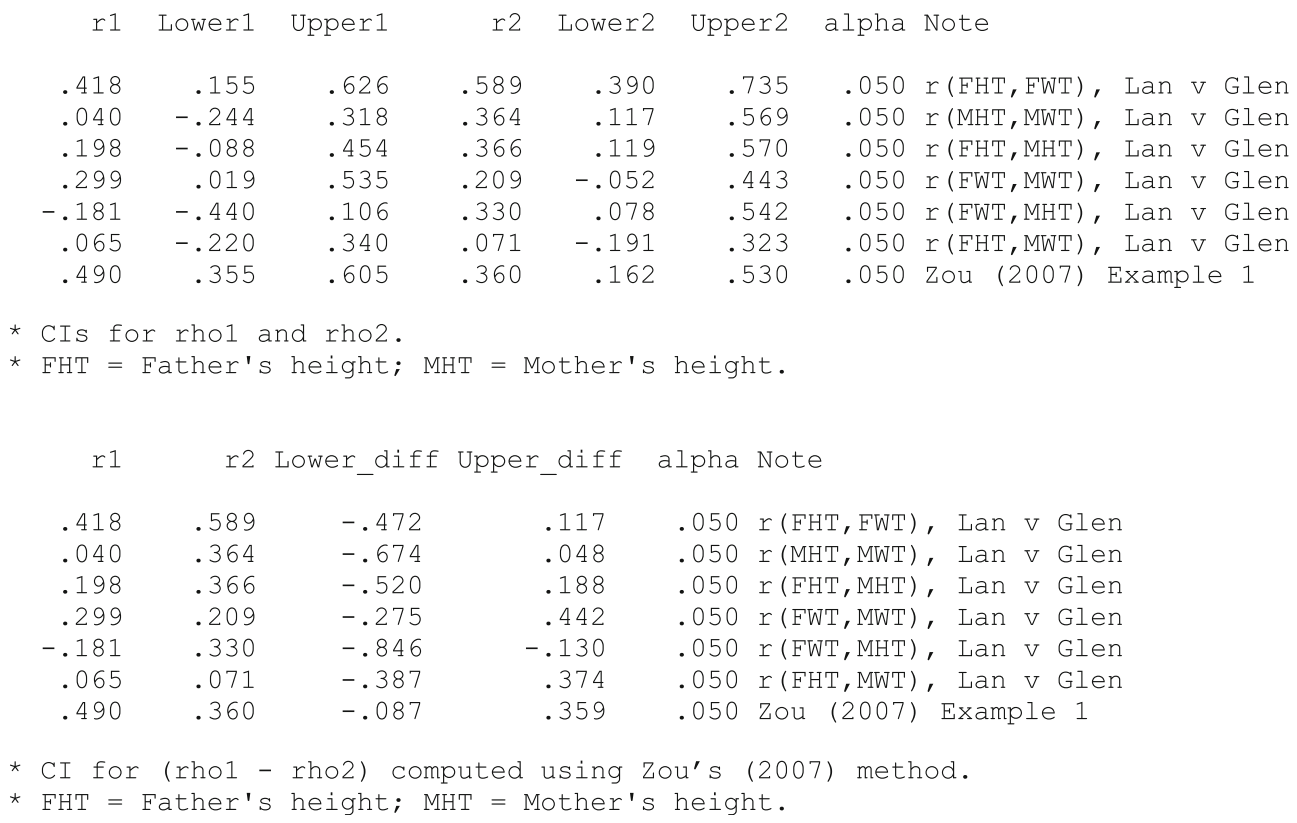

Testing the difference between two independent regression coefficients

If one has the results for OLS linear regression models from two independent samples, with the same criterion and explanatory variables used in both models, there may be some interest in testing the differences between corresponding coefficients in the two models. ${ }^{13}$ The required test is a simple extension of the $t$-test described earlier for testing the null hypothesis that $b^{*}=$ a specified value (see Eq. 8).

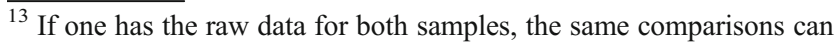
be achieved more directly by running a single model that uses all of the data and includes appropriate interaction terms. We will demonstrate that approach shortly.
} 
As was noted earlier, when one is dealing with two independent samples, the variance of a difference is the sum of the variances, and the standard error of the difference is the square root of that sum of variances. Therefore, the standard error of the difference between $b_{1}$ and $b_{2}$, two independent regression coefficients, is computed as shown in Eq. 12, where the two terms under the square root sign are the squares of the standard errors for $b_{1}$ and $b_{2}$. This standard error is used to compute the $t$-test shown in Eq. 13 and to compute the 100(1- $\alpha) \%$ CI (Eq. 14). The $t$-test has $d f=n_{1}+n_{2}-2 m-2$ (where $m=$ the common number of predictor variables in the two regression models, not including the constant). ${ }^{14}$ Some books (e.g., Howell, 2013) give the degrees of freedom for this $t$-test as $n_{1}+n_{2}-4$. That is because they are describing the special case where $m=1$ (i.e., the two regression models have only one predictor variable). And of course, $n_{1}+$ $n_{2}-2(1)-2=n_{1}+n_{2}-4$.

$$
\begin{aligned}
& s_{b_{1}-b_{2}}=\sqrt{s_{b_{1}}^{2}+s_{b_{2}}^{2}} \\
& t_{\left(n_{1}+n_{2}-2 m-2\right)}=\frac{b_{1}-b_{2}}{s_{b_{1}-b_{2}}} \\
& 100(1-\alpha) \% \text { CI for }\left(\mathrm{b}_{1}^{*}-\mathrm{b}_{2}^{*}\right) \\
& =\left(\mathrm{b}_{1}-\mathrm{b}_{2}\right) \pm \mathrm{t}_{\alpha / 2} \mathrm{~s}_{\mathrm{b}_{1}-\mathrm{b}_{2}} .
\end{aligned}
$$

To illustrate, we use the results for Lancaster and Glendora shown in Table 2 and also depicted graphically in Fig. 1. Specifically, we compare the regression coefficients (both intercept and slope) for Lancaster and Glendora. Plugging the coefficients and their standard errors (and sample sizes) into our code for Eq. 13, we get the output listed below:

$\begin{array}{rrrrrrrl}\text { b1 } & \text { b2 } & \text { bdiff } & \text { sediff } & t & \text { df } & p & \text { Note } \\ 148.053 & 130.445 & 17.608 & 15.125 & 1.164 & 103 & .247 & \text { Int, Lan v Glen } \\ 3.709 & 5.689 & -1.980 & 1.573 & -1.259 & 103 & .211 & \text { Slope, Lan v Glen }\end{array}$

The bdiff and sediff columns show the difference between the coefficients and the standard error of that difference - that is, the numerator and denominator of Eq. 13. Since both $p$ - values are greater than .05 , the null hypothesis cannot be rejected in either case. The next listing shows the CIs for bdiff. Because alpha $=.05$ on both lines of output, these are $95 \%$ CIs:

$\begin{array}{rrrrrrrl}\text { b1 } & \text { b2 } & \text { bdiff } & \text { sediff } & \text { alpha } \text { CI_Lower CI_Upper Note } \\ 148.053 & 130.445 & 17.608 & 15.125 & .050 & -12.388 & 47.604 \text { Int, Lan V Glen } \\ 3.709 & 5.689 & -1.980 & 1.573 & .050 & -5.100 & 1.140 \text { Slope, Lan v Glen }\end{array}$

The method we have just shown is fine in cases where one does not have access to the raw data but does have access to the required summary data. However, when the raw data are available, one can use another approach that provides more accurate results (because it eliminates rounding error). The approach we are referring to is sometimes called Potthoff analysis (see Potthoff, 1966). ${ }^{15}$ It entails running a hierarchical regression model. The first step includes only the predictor variable of primary interest (height in this case). On the second step, $k-1$ indicator variables are added to differentiate between the $k$ independent groups. The products of those indicators with the main

\footnotetext{
$\overline{{ }^{14} \text { In Eqs. } 12-14}$, the subscripts on $b_{1}$ and $b_{2}$ refer to which model the coefficients come from, not which explanatory variable they are associated with, as is typically done for models with two or more explanatory variables.
}

\footnotetext{
${ }^{15}$ Also see these unpublished documents on the second author's Web site: http://core.ecu.edu/psyc/wuenschk/docs30/CompareCorrCoeff.pdf, http://core.ecu.edu/psyc/wuenschk/MV/multReg/Potthoff.pdf).
} 
predictor variable are also added on step 2. In this case, we have $k=2$ groups (Lancaster and Glendora), so we add only one indicator variable and one product term on step 2. (We chose to use an indicator for area 2, Lancaster, thus making Glendora the reference category.) The SPSS commands to run this model were as follows, with fweight $=$ father's weight, fht $60=$ father's height centered on $60 \mathrm{in}$., $\mathrm{A} 2=$ an indicator for area 2 (Lancaster), and FHTxA2 = the product of fht60 and A2:

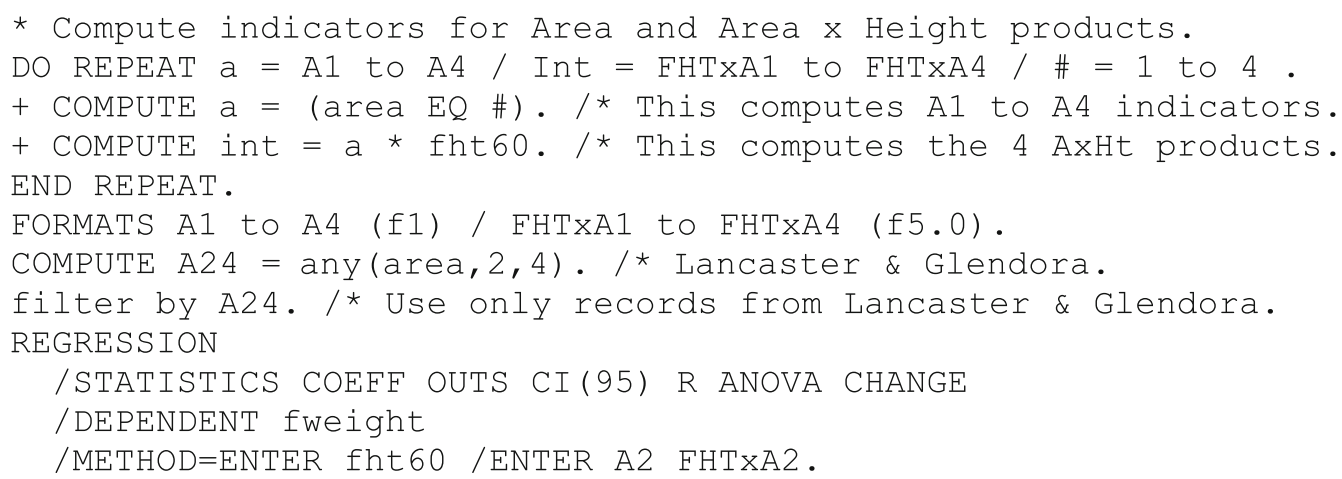

The $F$-test on the change in $R^{2}$ (from step 1 to step 2) tests the null hypothesis of coincidence, which states that the two population regression lines are identical (i.e., they have the same intercept and the same slope). In the table of coefficients for the full model (step 2), the $t$-test for the area 2 indicator variable tests the null hypothesis that the population intercepts are the same, and the $t$-test for the height $\times$ A2 product term tests the null hypothesis that the two population slopes are equal. (The $t$-test for height in the full model tests the null hypothesis that the population slope $=0$ for the reference group - that is, the group for which the area 2 indicator variable $=0$.)

We ran that hierarchical regression analysis for the Lancaster and Glendora data and found that the change in $R^{2}$ from step 1 to step $2=.011, F(2,103)=0.816, M S_{\text {residual }}=$ $44,362.179, p=.445$. Therefore, the null hypothesis of coincidence of the regression lines cannot be rejected. Normally, we would probably stop at this point, because there is no great need to compare the slopes and intercepts separately if we have already failed to reject the null hypothesis of coincident regression lines. However, in order to compare the results from this Potthoff analysis with results obtained earlier via Eq. 13, we shall proceed.

The regression coefficients for both steps of our hierarchical model are shown in Table 3. Looking at the step 2, the coefficient for the area 2 indicator is equal to the difference between the intercepts for Burbank and Glendora (see Table 2). The $t$ test for the area 2 indicator is not statistically significant, $t(103)$ $=1.168, p=.245$. Therefore, the null hypothesis that the two population intercepts are equal cannot be rejected. The coefficient for the height $\times$ A2 product term gives the difference between the slopes for Burbank and Glendora. The $t$-test for this product term is not statistically significant, $t(103)=-1.264$, $p=.209$. Therefore, the null hypothesis that the population slopes are the same cannot be rejected either. Finally, note that apart from rounding error, the results of these two tests match the results we got earlier by plugging summary data into Eq. 13: $t(103)=1.164, p=.247$, for the intercepts; and $t(103)=-1.259$, $p=.211$, for the slopes. (As has been noted, methods that use the raw data are generally preferred over methods that use summary data, because the former eliminate rounding error.)

\section{Methods for $\boldsymbol{k}$ independent parameters}

On occasion, one may wish to test a null hypothesis that says that three or more independent parameters are all equivalent. This can be done using the test of heterogeneity that is familiar to meta-analysts (see Fleiss, 1993, for more details). The test statistic is often called $Q^{16}$ and is computed as follows:

$Q=\sum_{i=1}^{k} W_{i}\left(Y_{i}-\bar{Y}\right)^{2}$,

where $k=$ the number of independent parameters, $Y_{i}=$ the estimate for the $i$ th parameter, $W_{i}=$ the reciprocal of its variance, and $\bar{Y}=$ a weighted average of the $k$ parameter estimates, which is computed as shown in Eq. 16. When the

\footnotetext{
${ }^{16}$ Meta-analysts often describe this statistic as Cochran's $Q$ and cite Cochran (1954). This may cause some confusion, however, because Cochran's $Q$ often refers to a different statistic used to compare $k$ related dichotomous variables, where $k \geq 3$. That test is described in Cochran (1950).
} 
Table 3 Parameter estimates for a hierarchical regression model with height entered on step 1 and an area 2 (Lancaster) indicator and its product with height both entered on step 2

Coefficient ${ }^{\mathrm{a}}$

\begin{tabular}{|c|c|c|c|c|c|c|c|c|}
\hline \multirow{2}{*}{\multicolumn{2}{|c|}{ Step }} & \multicolumn{2}{|c|}{ Unstandardized coefficients } & \multirow{2}{*}{$\begin{array}{l}\text { Standardized coefficients } \\
\text { Beta }\end{array}$} & \multirow[t]{2}{*}{$\mathrm{t}$} & \multirow[t]{2}{*}{ Sig. } & \multicolumn{2}{|c|}{$95.0 \%$ confidence interval for $\mathrm{B}$} \\
\hline & & $\mathrm{B}$ & Std. error & & & & Lower bound & Upper bound \\
\hline \multirow{2}{*}{1} & (Constant) & 138.793 & 7.510 & & 18.481 & .000 & 123.902 & 153.684 \\
\hline & Height of father (centered on 60 in) & 4.771 & .778 & .513 & 6.130 & .000 & 3.228 & 6.314 \\
\hline \multirow{4}{*}{$\begin{array}{r}2 \\
\\
\\
\end{array}$} & (Constant) & 130.445 & 10.511 & & 12.410 & .000 & 109.598 & 151.292 \\
\hline & Height of father (centered on 60 in) & 5.689 & 1.073 & .612 & 5.304 & .000 & 3.562 & 7.816 \\
\hline & Area 2 indicator & 17.608 & 15.075 & .367 & 1.168 & .245 & -12.289 & 47.505 \\
\hline & Height $\times$ A2 & -1.979 & 1.566 & -.403 & -1.264 & .209 & -5.086 & 1.127 \\
\hline
\end{tabular}

a. Dependent Variable: weight of father in pounds

null hypothesis is true (i.e., when all population parameters are equivalent), $Q$ is distributed (approximately) as chisquare with $d f=k-1$.

$\bar{Y}=\frac{\sum W_{i} Y_{i}}{\sum W_{i}}$

An example using regression coefficients

We illustrate this procedure using output from the four simple linear regression models summarized in Table 2. Using the method described above to test the null hypothesis that the four population intercepts are all the same, we get $Q$ $=1.479, d f=3, p=.687$. And testing the null hypothesis that the slopes are all the same, we get $Q=1.994, d f=3, p=$ .574. Therefore, we cannot reject the null hypothesis in either case.
Because the raw data are available in this case, we can also test the null hypothesis that all slopes are the same by performing another Potthoff analysis, like the one described earlier. With $k=4$ groups (or areas), we will need three (i.e., $k-1)$ indicator variables for area and three product terms. The test of coincidence will contrast the full model with a model containing only the continuous predictor variable (height). The test of intercepts will contrast the full model with a model from which the $k-1$ indicator variables have been removed. The test of slopes will contrast the full model with a model from which the $k-1$ interaction terms have been dropped.

Using SPSS, we ran a hierarchical regression model with height entered on step 1. On step 2, we added three indicators for area plus the products of those three indicators with height. The SPSS REGRESSION command for this analysis was as follows:

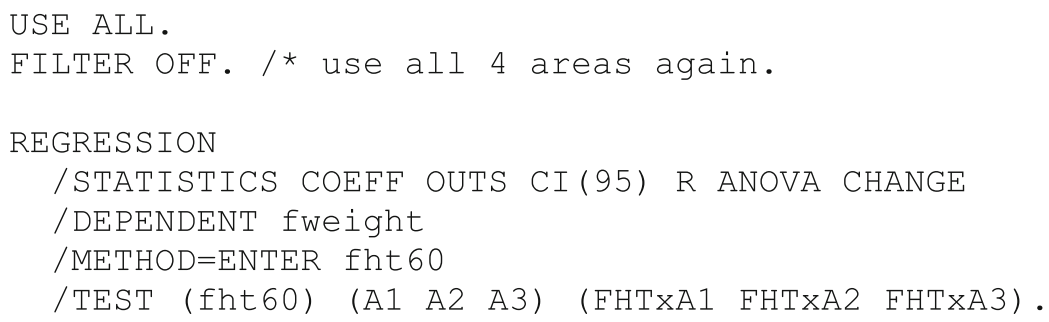

Table 4 shows the ANOVA summary table for this model, and Table 5 shows the parameter estimates. Because we used the TEST method (rather than the default ENTER method) for step 2 of the REGRESSION command, the ANOVA summary table includes the multiple degree of freedom tests we need to test the null hypotheses that all intercepts and all slopes are the same (see the "Subset Tests" section in Table 4). For SAS code that produces the same results, see the online supplementary material or the second author's Web site (http:/core.ecu.edu/ psyc/wuenschk/W\&W/W\&W-SAS.htm).

The $R^{2}$ values for steps 1 and 2 of our hierarchical regression model were .272 and .286 , respectively, and the 
Table 4 ANOVA summary table for the hierarchical regression model with height entered on step 1 and three area indicators and their products with height entered on step 2

\begin{tabular}{|c|c|c|c|c|c|c|c|c|}
\hline \multicolumn{9}{|c|}{ ANOVA $^{\mathrm{d}}$} \\
\hline \multicolumn{3}{|c|}{ Step } & \multirow{2}{*}{$\frac{\text { Sum of squares }}{23221.739}$} & \multirow{2}{*}{$\frac{\mathrm{df}}{1}$} & \multirow{2}{*}{$\begin{array}{c}\text { Mean square } \\
23221.739\end{array}$} & \multirow{2}{*}{$\begin{array}{l}\mathrm{F} \\
55.189\end{array}$} & \multirow{2}{*}{$\begin{array}{l}\text { Sig. } \\
.000^{\mathrm{a}}\end{array}$} & \multirow[t]{2}{*}{ R square change } \\
\hline 1 & Regression & & & & & & & \\
\hline & Residual & & 62274.134 & 148 & 420.771 & & & \\
\hline & Total & & 85495.873 & 149 & & & & \\
\hline \multirow[t]{6}{*}{2} & Subset Tests & Height of father (centered on 60 in) & 12117.765 & 1 & 12117.765 & 28.182 & $.000^{\mathrm{b}}$ & .142 \\
\hline & & Area 1 indicator, Area 2 indicator, Area 3 indicator & 631.267 & 3 & 210.422 & .489 & $.690^{\mathrm{b}}$ & .007 \\
\hline & & Height $\times$ A1, Height $\times$ A2, Height $\times$ A3 & 850.075 & 3 & 283.358 & 659 & $.579^{\mathrm{b}}$ & .010 \\
\hline & Regression & & 24438.781 & 7 & 3491.254 & 8.120 & $.000^{\mathrm{c}}$ & \\
\hline & Residual & & 61057.092 & 142 & 429.980 & & & \\
\hline & Total & & 85495.873 & 149 & & & & \\
\hline
\end{tabular}

a. Predictors: (Constant), Height of father (centered on 60 in)

b. Tested against the full model

c. Predictors in the Full Model: (Constant), Height of father (centered on 60 in), Area 3 indicator, Area 1 indicator, Height $\times$ A2, Height $\times$ A1, Height $\times$ A3, Area 2 indicator

d. Dependent Variable: weight of father in pounds

change in $R^{2}$ from step 1 to 2 was equal to $.014, F(6$, 142) $=0.472, p=.828 .{ }^{17}$ Therefore, the null hypothesis of coincident regression lines cannot be rejected. Nevertheless, we shall report separate tests for the intercepts and slopes in order to compare the results from this analysis with those we obtained earlier via Eq. 15. The test for homogeneity of the intercepts is the second Subset Test in Table 4-that is, the combined test for the area 1 , area 2 , and area 3 indicators. It shows that the null hypothesis of homogeneous intercepts cannot be rejected, $F(3,142)=0.489, p=.690$. When testing this same hypothesis via Eq. 15 , we got $Q=1.479, d f=3$, $p=.687$. The test of homogeneity of the slopes in the Potthoff analysis is the third Subset Test in Table 4that is, the combined test for the three product terms. It shows that the null hypothesis of homogeneous slopes cannot be rejected, $F(3,142)=0.659, p=.579$. Earlier, using Eq. 15 , we got $Q=1.994, d f=3, p=.574$, when testing for homogeneity of the slopes.

Note that for both of these tests, the $p$-values for the $Q$ and the $F$-tests are very similar. The differences are partly due to rounding error in the computation of $Q$ (where we rounded the coefficients and their standard errors to three decimals) and partly due to the fact that the denominator degrees of freedom for the $F$-tests are less than infinite. For

\footnotetext{
$\overline{17}$ The three "R Square Change" values in Table 4 give the change in $R^{2}$ for removal of each of the three subsets of predictors from the final (full) model. They do not give the change in $R^{2}$ from step 1 to step 2 of the hierarchical model.
}

a good discussion of the relationship between $F$ and $\chi^{2}$ tests (bearing in mind that $Q$ is approximately distributed as $\chi^{2}$ when the null hypothesis is true), see Gould's (2009) post on the Stata FAQ Web site (http://www.stata.com/support/faqs/ stat/wald.html).

Finally, we should clarify how the coefficients and $t$ tests for the full model (Table 5, step 2) are interpreted. The intercept for the full model is equal to the intercept for area 4 (Glendora), the omitted reference group (see Table 2 for confirmation). The coefficients for the three area indicators give the differences in intercepts between each of the other three areas and area 4 (with the area 4 intercept subtracted from the other intercept in each case). None of those pairwise comparisons are statistically significant (all $p$-values $\geq .244$ ). The coefficient for height gives the slope for area 4 , and the coefficients for the three product terms give differences in slope between each of the other areas and area 4 (with the area 4 slope subtracted from the other slope). None of the pairwise comparisons for slope are statistically significant either (all $p$-values $\geq .208$ ).

An example using correlation coefficients

When using the test of heterogeneity with correlations, it is advisable to first apply Fisher's $r$-to- $z$ transformation. To illustrate, we use the correlation between father's height and father's weight in Table 1. The values of that correlation in the four areas were .628 , $.418, .438$, and .589 (with sample sizes of $24,49,19$, and 58, respectively). The $r^{\prime}$ values for these 
Table 5 Parameter estimates for a hierarchical regression model with height entered on step 1 and three area indicators and their products with height entered on step 2

\begin{tabular}{|c|c|c|c|c|c|c|c|}
\hline \multirow[t]{2}{*}{ Step } & \multicolumn{2}{|c|}{ Unstandardized coefficients } & \multirow{2}{*}{$\begin{array}{l}\text { Standardized coefficients } \\
\text { Beta }\end{array}$} & \multirow[t]{2}{*}{$\mathrm{t}$} & \multirow[t]{2}{*}{ Sig. } & \multicolumn{2}{|c|}{$95.0 \%$ confidence interval for $\mathrm{B}$} \\
\hline & $\mathrm{B}$ & Std. error & & & & Lower bound & Upper bound \\
\hline 1 (Constant) & 140.491 & 5.844 & & 24.039 & .000 & 128.942 & 152.040 \\
\hline Height of father (centered on 60 in) & 4.492 & .605 & .521 & 7.429 & .000 & 3.297 & 5.687 \\
\hline 2 (Constant) & 130.445 & 10.502 & & 12.420 & .000 & 109.684 & 151.206 \\
\hline Height of father (centered on 60 in) & 5.689 & 1.072 & .660 & 5.309 & .000 & 3.570 & 7.807 \\
\hline Area 1 indicator & 11.566 & 15.758 & .178 & .734 & .464 & -19.584 & 42.717 \\
\hline Area 2 indicator & 17.608 & 15.062 & .346 & 1.169 & .244 & -12.167 & 47.383 \\
\hline Area 3 indicator & 13.593 & 19.591 & .189 & .694 & .489 & -25.135 & 52.321 \\
\hline Height $\times$ A1 & -1.510 & 1.622 & -.226 & -.931 & .354 & -4.716 & 1.697 \\
\hline Height $\times$ A2 & -1.979 & 1.565 & -.375 & -1.265 & .208 & -5.073 & 1.114 \\
\hline Height $\times$ A3 & -1.940 & 2.002 & -.266 & -.969 & .334 & -5.897 & 2.017 \\
\hline
\end{tabular}

a. Dependent Variable: weight of father in pounds

correlations are $.7381, .4453, .4698$, and .6761 . These are the $Y_{i}$ values we will use in Eqs. 15 and 16. The variance of the sampling distribution of $r^{\prime}$ is equal to $1 /(n-3)$, so the $W_{i}$ values needed in Eqs. 15 and 16 are simply $n_{i}-3$ (i.e., $21,46,16$, and 55). Plugging these $W_{i}$ and $Y_{i}$ values into Eq. 16 yields $\bar{Y}$ equal to .5847. Solving Eq. 15 for these data results in $Q=$ 2.060, $d f=3, p=.560$. Therefore, the null hypothesis that the four population correlations are equal cannot be rejected.

Finally, we should point out that when the procedure described here is used to test the equivalence of two correlations, the result is identical to that obtained via the $z$-test for comparing two independent correlations $\left(z^{2}=Q\right)$. For example, when we used this procedure to compare the correlation between father's weight and mother's height for Lancaster, $r=-.181, n=49, p=$ .214 , with the same correlation for Glendora, $r=.330$, $n=58, p=.011$, we got $Q=6.927, d f=1, p=.008$. Comparing these same two correlations earlier using Eq. 11, we got $z=-2.632, p=.008$.

\section{Methods for two nonindependent parameters}

In this section, we describe two standard methods for comparing two nonindependent correlations. These methods are applicable when both of the correlations to be compared have been computed using the same sample. One method is for the situation where the two correlations have a variable in common (e.g., $r_{12}$ vs. $\left.r_{13}\right)$, and the other for the situation where there are no variables in common (e.g., $r_{12}$ vs. $r_{34}$ ). (The first situation is sometimes described as overlapping, and the second as nonoverlapping.)

Two nonindependent correlations with a variable in common

Hotelling (1931) devised a test for comparing two nonindependent correlations that have a variable in common, but Williams (1959) came up with a better test, which is still in use today. Although Williams actually described it as an $F$-test, it is more commonly presented as a $t$-test nowadays. ${ }^{18}$ Equation 17 shows the formula for Williams's $t$-test:

$$
\begin{aligned}
& t_{n-3}=\left(r_{12}-r_{13}\right) \sqrt{\frac{(n-1)\left(1+r_{23}\right)}{2\left(\frac{n-1}{n-3}\right)|R|+\frac{\left(r_{12}+r_{13}\right)^{2}}{4}\left(1-r_{23}\right)^{3}}} \\
& \text { where } \quad|\mathrm{R}|=1-\mathrm{r}_{12}^{2}-\mathrm{r}_{13}^{2}-\mathrm{r}_{23}^{2}+2 \mathrm{r}_{12} \mathrm{r}_{13} \mathrm{r}_{23}
\end{aligned}
$$

To illustrate Williams's (1959) test, we use correlations reported in Table 1. Within each of the four

\footnotetext{
$\overline{18}$ Because Williams's (1959) test statistic was distributed (approximately) as $F$, with $d f=1$ and $n-3$, its square root is distributed (approximately) as $t$ with $d f=n-3$.
} 
areas, we wish to compare $r_{12}$ and $r_{13}$, with $X_{1}=$ father's height, $X_{2}=$ mother's height, and $X_{3}=$ mother's weight. Thus, the comparisons we wish to make are as follows: .164 vs. -.189 (Burbank), .198 vs. .065 (Lancaster), .412 vs. .114 (Long Beach), and .366 vs. .071 (Glendora). The $r_{23}$ values for the four areas (i.e., the correlations between mother's height and mother's weight) are $.624, .040, .487$, and .364, respectively. Plugging the appropriate values into Eq. 17 yields the results listed below. The CI included in the results is a CI on $\rho_{12}-\rho_{13}$ computed using Zou's (2007) modified asymptotic method. (Zou's example 2 is included in order to confirm that our code produces his result.)

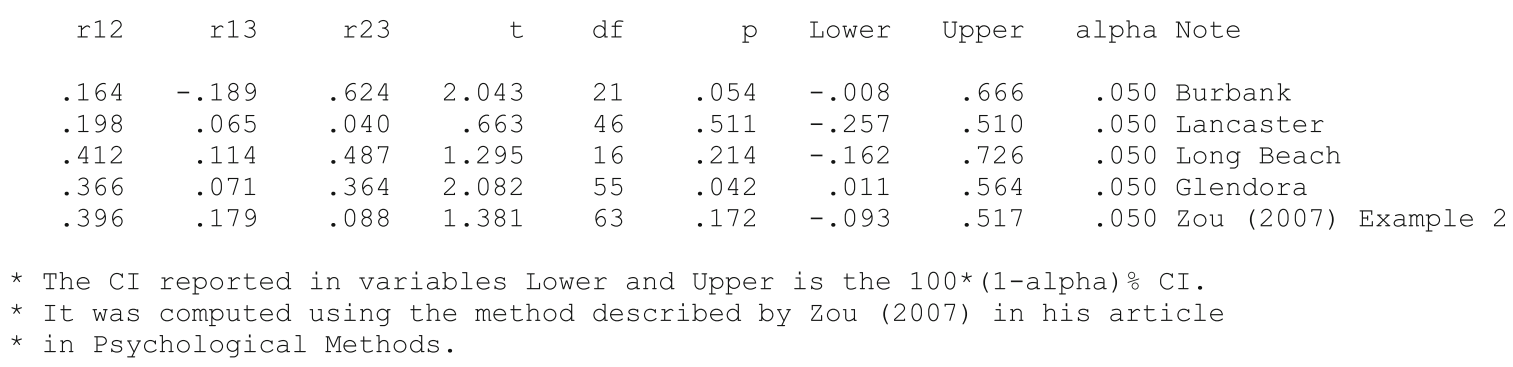

These results indicate that the difference between the two correlated correlations is statistically significant only in area 4 , Glendora, $t_{55}=2.082, p=.042$. As was expected, that is also the only case in which the $95 \%$ CI for $\rho_{12}-\rho_{13}$ does not include 0 .

Two nonindependent correlations with no variables in common

Pearson and Filon (1898) devised a method for comparing two nonindependent correlations with no variables in common, but a revised version of it by Steiger (1980) yields a "theoretically better test statistic" (Raghunathan et al., 1996, p. 179). Pearson and Filon's original statistic is often called PF and is calculated as shown in Eq. 18.

$$
\begin{aligned}
& P F=\frac{r_{12}-r_{34}}{s_{r_{12}-r_{34}}}=\frac{r_{12}-r_{34}}{\sqrt{\frac{\left(1-r_{12}^{2}\right)^{2}+\left(1-r_{34}^{2}\right)^{2}-k}{n}}} \\
& \text { where } \\
& \quad k=\left(r_{13}-r_{23} r_{12}\right)\left(r_{24}-r_{23} r_{34}\right) \\
& \quad+\left(r_{14}-r_{13} r_{34}\right)\left(r_{23}-r_{13} r_{12}\right) \\
& \quad+\left(r_{13}-r_{14} r_{34}\right)\left(r_{24}-r_{14} r_{12}\right) \\
& \quad+\left(r_{14}-r_{12} r_{24}\right)\left(r_{23}-r_{24} r_{34}\right)
\end{aligned}
$$

The modified version of the Pearson-Filon statistic, which is usually called $Z P F$, can be calculated using Eq. 19. The $Z$ in $Z P F$ is there because this statistic is calculated using $r^{\prime}$ values (obtained via Fisher's $r$-to- $z$ transformation) in the numerator ${ }^{19}$ :

$$
Z P F=\frac{r_{12}^{\prime}-r_{34}^{\prime}}{s_{r_{12}^{\prime}-r_{34}^{\prime}}^{\prime}}=\frac{r_{12}^{\prime}-r_{34}^{\prime}}{\sqrt{\left(1-\frac{k}{2\left(1-r_{12}^{2}\right)\left(1-r_{34}^{2}\right)}\right)\left(\frac{2}{n-3}\right)}}
$$

To illustrate this method, let $r_{12}=$ the correlation between father's height and weight and $r_{34}$ the correlation between mother's height and weight and compare $r_{12}$ and $r_{34}$ in each of the four areas separately, but also for all of the data, collapsing across area. ${ }^{20}$ The correlations within each area are shown in Table 1. Collapsing across area, the correlation between height and weight is .521 $(p<.001)$ for fathers and .318 $(p<.001)$ for mothers, with $n=150$ for both. Plugging those values into Eq. 18 yields the results shown

\footnotetext{
${ }^{19}$ The reason this statistic is called $Z P F$ is that Fisher used $\mathrm{z}$ to symbolize correlations that had been transformed using his $r$-to- $z$ transformation. As was noted earlier, many current authors use $r^{\prime}$ rather than $z$, to avoid confusion with $z$-scores or $z$-test values.

${ }^{20}$ As was noted earlier, the lung function data file has matched pairs of fathers and mothers, which is why the correlation between height and weight for fathers is not independent of the same correlation for mothers.
} 
below. The $100 \times(1-\alpha) \%$ CI shown in these results was computed using Zou's (2007) method. (Zou's third example was included to ensure that his method has been implemented correctly in our code.)

\begin{tabular}{|c|c|c|c|c|c|c|c|c|c|}
\hline r12 & r34 & $\mathrm{PF}$ & $\mathrm{ZPF}$ & $P_{-} \mathrm{PF}$ & P_ZPF & Lower & Upper & alpha & Note \\
\hline .628 & .624 & .023 & .022 & .982 & .983 & -.373 & .382 & .050 & $\mathrm{~F} \vee \mathrm{M}, \mathrm{Bur}$ \\
\hline .418 & .040 & 2.129 & 2.027 & .033 & .043 & .011 & .716 & .050 & $\mathrm{~F} V \mathrm{M}, \mathrm{Lan}$ \\
\hline .438 & .487 & -.208 & -.191 & .835 & .848 & -.550 & .452 & .050 & F V M, L Beach \\
\hline .589 & .364 & 1.614 & 1.582 & .107 & .114 & -.054 & .507 & .050 & $\mathrm{~F} \vee \mathrm{M}, \mathrm{Glen}$ \\
\hline .521 & .316 & 2.255 & 2.236 & .024 & .025 & .025 & .384 & .050 & $\mathrm{~F} V \mathrm{M}, \mathrm{All}$ \\
\hline .396 & .189 & 1.375 & 1.338 & .169 & .181 & -.096 & .501 & .050 & Zou Example 3 \\
\hline
\end{tabular}

The $P F$ and $Z P F$ columns show the Pearson-Filon and modified Pearson-Filon statistics, respectively, and the $p_{-} P F$ and $p_{-} Z P F$ columns show the corresponding $p$-values. Thus, the difference between the two correlated correlations is statistically significant only for the sample from Lancaster ( $p$ for $Z P F=.043)$ and for the analysis that uses data from all four areas $(p$ for $Z P F=.025)$. Because alpha $=.05$ on all rows, all CIs are $95 \%$ CIs.

\section{Summary}

Our goal in writing this article was twofold. First, we wished to provide, in a single resource, descriptions and examples of the most common procedures for statistically comparing Pearson correlations and regression coefficients from OLS models. All of these methods have been described elsewhere in the literature, but we are not aware of any single book or article that discusses all of them. In the past, therefore, researchers or students who have used these tests may have needed to track down several resources to find all of the required information. In the future, by way of contrast, they will be able to find all of the required information in this one article.

Our second goal was to provide actual code for carrying out the tests and computing the corresponding $100 \times(1-\alpha)$ CIs, where applicable. ${ }^{21}$ Most if not all of the books and articles that describe these tests (including our own article) present formulae. But more often than not, it is left to readers to translate those formulae into code. For people who are well-versed in programming, that may not present much of a challenge. However, many students and researchers are not

\footnotetext{
${ }^{21}$ Although we provide code for SPSS and SAS only, users of other statistics packages may also find it useful, since there are many commonalities across packages. For example, the first author was able to translate SAS code for certain tests into SPSS syntax without difficulty, and the second author was able to translate in the opposite direction without difficulty.
}

well-versed in programming. Therefore, their attempts to translate formulae into code are liable to be very time consuming and error prone, particularly when they are translating some of the more complicated formulae (e.g., Eq. 17 in the present article).

Finally, we must acknowledge that resampling methods provide another means of comparing correlations and regression coefficients. For example, Beasley et al. (2007) described two bootstrap methods for testing a null hypothesis that specifies a nonzero population correlation. Such methods are particularly attractive when distribution assumptions for asymptotic methods are too severely violated or when sample sizes are small. However, such methods cannot be used if one has only summary data; they require the raw data. Fortunately, in many cases, the standard methods we present here do work quite well, particularly when the samples are not too small.

In closing, we hope that this article and the code that accompanies it will prove to be useful resources for students and researchers wishing to test hypotheses about Pearson correlations or regression coefficients from OLS models or to compute the corresponding CIs.

Acknowledgments We thank Dr. John Jamieson for suggesting that an article of this nature would be useful to researchers and students. We thank Drs. Abdelmonem A. Afifi, Virginia A. Clark, and Susanne May for allowing us to include their lung function data set with this article. And finally, we thank three anonymous reviewers for their helpful comments on an earlier draft of the manuscript.

Competing interests None of the authors have any competing interests.

\section{References}

Afifi, A. A., Clark, V., \& May, S. (2003). Computer-aided multivariate analysis (4th Ed.). London, UK: Chapman \& Hall/CRC. (ISBN10: 1584883081; ISBN-13: 978-1584883081). 
Beasley, W. H., DeShea, L., Toothaker, L. E., Mendoza, J. L., Bard, D. E., \& Rodgers, J. L. (2007). Bootstrapping to test for nonzero population correlation coefficients using univariate sampling. Psychological Methods, 12, 414-433.

Cochran, W. G. (1950). The comparison of percentages in matched samples. Biometrika, 37, 256-266.

Cochran, W. G. (1954). The combination of estimates from different experiments. Biometrics, 10, 101-129.

Fisher, R. A. (1921). On the probable error of a coefficient of correlation deduced from a small sample. Metron, 1, 3-32.

Fleiss, J. L. (1993). The statistical basis of meta-analysis. Statistical Methods in Medical Research, 2, 121-145.

Gould, W. (2009, July). Why does test sometimes produce chi-squared and other times $F$ statistics? how are the chi-squared and $F$ distributions related? [Support FAQ] Retrieved from http:// www.stata.com/support/faqs/statistics/chi-squared-and-fdistributions/

Hotelling, H. (1931). The generalization of Student's ratio. Annals of Mathematical Statistics, 2, 360-378.

Howell, D. C. (2013). Statistical methods for psychology (8th ed.). Belmont, CA: Cengage Wadsworth.
Kenny, D. A. (1987). Statistics for the social and behavioral sciences. Boston, MA: Little, Brown and Company.

Pearson, K., \& Filon, L. G. N. (1898). Mathematical contributions to the theory of evolution. IV. On the probable errors of frequency constants and on the influence of random selection on variation and correlation. Transactions of the Royal Society London (Series A), 191, 229-311.

Potthoff, R. F. (1966). Statistical aspects of the problem of biases in psychological tests. (Institute of Statistics Mimeo Series No. 479). Chapel Hill: University of North Carolina, Department of Statistics. URL: http://www.stat.ncsu.edu/information/library/mimeo. archive/ISMS_1966_479.pdf

Raghunathan, T. E., Rosenthal, R., \& Rubin, D. B. (1996). Comparing correlated but nonoverlapping correlations. Psychological Methods, 1, 178-183.

Steiger, J. H. (1980). Tests for comparing elements of a correlation matrix. Psychological Bulletin, 87, 245-251.

Williams, E. J. (1959). The comparison of regression variables. Journal of the Royal Statistical Society (Series B), 21, 396-399.

Zou, G. Y. (2007). Toward using confidence intervals to compare correlations. Psychological Methods, 12, 399-413. 